\title{
Can You Hear Me Now? Telemedicine in Rural America
}

\author{
Jerome C Siy, MD, MHA, SFHM*, Ameet Doshi, MD, MBA
}

Department of Hospital Medicine, HealthPartners, Bloomington, Minnesota.

ommon themes run through rural communities and their health needs, yet the rurality of our nation is quite diverse. Approximately $97 \%$ of the United States is rural, and yet there is no silver bullet to resolve the health disparities that exist between urban and rural America. Differences in economic condition, infrastructure, education, racial diversity, health habits, and job opportunities contribute to the health disparities seen in rural communities.

In this issue of the Journal of Hospital Medicine, Gutierrez and colleagues ${ }^{1}$ evaluate the implementation and outcomes of a rural telehospitalist program. In a hub-and-spoke fashion, providers at a large tertiary care hospital utilized telemedicine to round on patients with an onsite advanced practice provider at a 10-bed critical access hospital. Outcomes were examined during pre- and postimplementation periods using quantitative metrics (length of stay [LOS], readmission rate, mortality, and satisfaction) and qualitative measures based on interviews with staff. LOS was reduced in the postimplementation period, with a lower but nonsignificant readmission rate and no difference in mortality. Overall satisfaction was high, although respondents noted significant communication and technology issues. This study helps broaden telemedicine research and opens the conversation on barriers in rural implementation.

As we increasingly focus on the provision and financing of care aimed at the health of populations, the diverse issues facing rural America remain insufficiently addressed. Probst and colleagues ${ }^{2}$ suggest that population-based health policies are biased toward large urban centers. Innovations to transcend this "structural urbanism" are still fraught with obstacles, as revealed during the 2020 public health emergency (PHE). Telemedicine, once thought the solution to rural health provider shortages, has not escaped structural urbanism. Prior to the PHE, Medicare did not cover many core services delivered via telehealth, including hospitalist service codes. Waivers during the PHE have temporarily opened up reimbursement pathways. Unlike most telehospitalist services, Gutierrez and colleagues did not face these payment barriers in their study. Without claims data to inform payors on the adequacy of such services, health systems remain unable to promote telemedicine as a solution to rural access and cost issues. For example, in 2018, Yu and colleagues ${ }^{3}$ described the state of telemedicine in Minnesota, again, without claims data to inform supply or demand for hospitalist services.

*Corresponding Author: Jerome C. Siy, MD, MHA;

Email: jerome.c.siy@healthpartners.com; Telephone: 952-883-6181; Twitter: @jcsiy.

Received: October 24, 2020; Accepted: November 12, 2020

(c) 2021 Society of Hospital Medicine DOI 10.12788/jhm.3569
Beyond payment barriers, technology issues are a challenge to rural telemedicine. Access to affordable, simple, and reliable equipment and broadband internet is key to user satisfaction. In April 2020, the Federal Communications Commission estimated that 18 million Americans had insufficient access to broadband internet. ${ }^{4}$ This number points to the technological hurdles of rural telemedicine.

Medicine, a highly relationship-based "team sport," is another barrier to successful implementation. Whether serving as supervisor or primary attending (as noted in the evaluation by JaKa and colleagues ${ }^{5}$ ), the telehospitalist must be "on stage" for both patients and remote spoke colleagues. In our experience, telehospitalists also practice in-person daytime hospital medicine at spoke sites; this further enhances their relationship and connection with the spoke community and likely contributes to high satisfaction by hub hospitalists and spoke patients and nurses.

These factors create a clear impetus for further evaluation of rural telemedicine programs. There is an extensive range of program structures to evaluate, from cross-coverage to a 24/7 virtual hospital. Satisfaction is also relative and must contextualize quantitative measurement to historic care models. The execution of a telehospitalist program should align with the goals and objectives of spoke hospitals, as satisfaction will reflect how well hub hospitalists are able to meet those needs. Thus, multicenter studies that examine commercial financial pressures and encompass a variety of patient populations are imperative.

A hub-and-spoke telemedicine program can be a crucial resource for rural hospitals. The foundation of this model revolves around key factors, including reliable financing, access to technology, seamless communication, and engendering satisfaction among providers, staff, and patients. Research must continue for these programs to overcome the financial and structural challenges to their success.

Disclosures: The authors have no potential conflicts of interest to disclose.

\section{References}

1. Gutierrez J, Moeckli J, Holcombe A, et al. Implementing a telehospitalist program between Veterans Health Administration hospitals: outcomes, acceptance, and barriers to implementation. J Hosp Med. 2021;16:156-163. https://doi.org/10.12788/jhm.3570

2. Probst J, Eberth JM, Crouch, E. Structural urbanism contributes to poorer health outcomes for rural America. Health Aff (Millwood). 2019;38(12):19761984. https://doi.org/10.1377/hlthaff.2019.00914

3. Yu J, Mink P, Huckfeldt PJ, Guildemeister S, Abraham JM. Population-level estimates of telemedicine service provision using an all-payer claims database. Health Aff (Millwood). 2018;37(12):1931-1939. https://doi.org/10.1377/ hlthaff.2018.05116

4. Federal Communications Commission. (2020). 2020 Broadband Deployment Report. Washington, DC: Federal Communications Commission.

5. JaKa MM, Dinh JM, Ziegenfuss JY, et al. Patient and care team perspectives of telemedicine in critical access hospitals. J Hosp Med. 2020;15(6):345-348. https://doi.org/10.12788/jhm.3412 